\title{
Prognostic value of complete remission with superior platelet counts in acute myeloid leukemia
}

Abhishek Mangaonkar, MBBS, ${ }^{1}$ Hongyan $\mathrm{Xu}, \mathrm{PhD},{ }^{2}$ Jamal Mohsin, $\mathrm{MD},{ }^{1}$ Joshua Mansour, MD, ${ }^{1}$ Rohini Chintalapally, MBBS, ${ }^{1}$ Ryan Keen, MD,${ }^{1}$ Ashis K Mondal, PhD, ${ }^{3}$ David DeRemer, Pharm D, ${ }^{4,5}$ Amber B Clemmons, Pharm D, ${ }^{4,5}$ Stephen M Clark, Pharm D, ${ }^{4,5}$ Arpita Shah, Pharm D, ${ }^{4,5}$ Anand Jillela, MBBS,${ }^{6}$ Ravindra Kolhe, MBBS, PhD, ${ }^{3}$ and Vamsi Kota, MBBS $^{6}$

Departments of ${ }^{1}$ Internal Medicine, ${ }^{2}$ Biostatistics, ${ }^{3}$ Pathology, and ${ }^{4}$ Pharmacy, Medical College of Georgia, Augusta, Georgia; ${ }^{5}$ Department of Clinical and Administrative Pharmacy, University of Georgia College of Pharmacy, Augusta; and ${ }^{6}$ Winship Cancer Institute of Emory University, Atlanta, Georgia

Background Complete remission (CR) in acute myeloid leukemia (AML) is defined as having $\leq 5 \%$ leukemic blast cells in the bone marrow and return of normal hematopoiesis after the first induction cycle. There is a subset of patients, however, who achieve reduction of leukemic blast cells with a subnormal platelet count, designated as CR with incomplete platelet recovery (platelet count, $\leq 100,000 / \mathrm{mcL}$; normal, 150,000-450,000/mcL), which is associated with inferior outcomes when compared with CR. Furthermore, there is another subset of patients with CR but superior platelet counts $(\geq 400,000 / \mathrm{mcl})$ whose prognostic significance is unclear.

Objective To establish whether CR with superior platelet counts is associated with better outcomes and can be used as a separate entity for prognostication.

Methods A retrospective chart review of 104 cases of AML was conducted. The highest platelet count during days 25-35 from initiation of induction chemotherapy (designated as day 30 platelet count) was documented. A multivariate analysis for other factors such as age, sex, risk categories, day 14+ plasma cell count (average plasma cell percentage at days 14-21), infections, allogeneic bone marrow transplant, and remission status was done.

Results Day 30 platelet count was found to be an independent predictor of survival in AML. On the multivariate analysis, the subgroup with superior platelet counts $(\geq 400,000 / \mathrm{mcL})$ was found to be associated with better outcomes.

Limitations Results need to be validated in a larger cohort.

Conclusions CR with superior platelet recovery $(\geq 400,000 / \mathrm{mcL})$ is a unique subcategory in itself and has prognostic significance. This may help better assess response to chemotherapeutic agents and aid in further decision-making regarding treatment.

I n 2014, there were about 18,860 new cases of adult acute myeloid leukemia (AML) and 10,460 deaths from the disease in the United States, which is more than any other type of leukemia. ${ }^{1}$ It is the sixth most common cancer in men and women. ${ }^{1}$ Therapeutic advances in AML have resulted in improved complete remission (CR) rates $;^{1}$ however, there are still a number of aspects of this disease for which evidence-based guidelines are lacking, such as induction therapy in older patients as well as postremission therapy in all patients. ${ }^{2}$ It is important to have an understanding of short- and long-term outcomes of treatment regimens because most of these therapies have serious, often debilitat- ing adverse effects. Hence, individualizing therapy in patients using a risk-benefit model would help in choosing appropriate regimens. Emerging data has identified various cytogenetics and somatic mutations with prognostic value in AML. This has broadened our understanding of the disease, in particular its heterogeneity; however, the impact of most of these genetic and molecular mutations on clinical practice remains unclear. ${ }^{3}$ There is still a need for development of an accurate and personalized risk prediction model for patients to individualize therapies, especially in the elderly population.

Chemotherapy with a cytarabine plus anthracycline (doxorubicin or daunorubicin) combination

Accepted for publication December 21, 2015. Correspondence: Abhishek Mangaonkar, MBBS; amangaonkar@gru.edu. Disclosures: The authors report no disclosures or conflicts of interest. JCSO 2016;14:66-71. (22016 Frontline Medical Communications. doi:10.12788/jicso.0231. 
TABLE 1 Baseline patient characteristics ( $N=104$

\section{Characteristic}

\begin{tabular}{|c|c|}
\hline \multicolumn{2}{|l|}{ Age, y } \\
\hline$\leq 55$ & $43(41.3)$ \\
\hline$>56-74$ & $53(51)$ \\
\hline$\geq 75$ & 8 (7.7) \\
\hline \multicolumn{2}{|l|}{ Sex } \\
\hline Male & $54(52)$ \\
\hline Female & $50(48)$ \\
\hline \multicolumn{2}{|l|}{ Race } \\
\hline White & $65(62.5)$ \\
\hline African-American & $35(33.7)$ \\
\hline Hispanic & $3(2.9)$ \\
\hline Unknown & $1(0.9)$ \\
\hline \multicolumn{2}{|l|}{ Risk $^{a}$} \\
\hline Favorable & $13(12.5)$ \\
\hline Intermediate & $59(56.73)$ \\
\hline Adverse & $21(20.19)$ \\
\hline Unknown & $11(10.58)$ \\
\hline \multicolumn{2}{|l|}{ Culture-proven infection ${ }^{b}$} \\
\hline$\leq 1$ month before induction & $1(1)$ \\
\hline During induction ${ }^{c}$ & $24(23.1)$ \\
\hline$\leq 6$ months after induction & $22(21.1)$ \\
\hline No infection during cycle & $57(54.8)$ \\
\hline \multicolumn{2}{|l|}{ Remission } \\
\hline Yes & $63(60.6)$ \\
\hline No & $30(28.8)$ \\
\hline Unknown & $11(10.6)$ \\
\hline \multicolumn{2}{|l|}{ Allogenic SCT } \\
\hline Yes & $16(15.4)$ \\
\hline No & $88(84.6)$ \\
\hline
\end{tabular}

aBased on cytogenetics/molecular analysis as per the European LeukemiaNet recommendations ${ }^{8}$ (see Figure 3). ${ }^{b}$ Culture-proven infections in relation to the first cycle of induction chemotherapy. 'Days 0-30 of chemotherapy.

(7+3 regimen) has resulted in a CR rate of between 50\%$70 \%$ in $\mathrm{AML}$ patients younger than 60 years, although rates are variable for elderly patients. ${ }^{4-7} \mathrm{CR}$ is commonly defined as bone marrow blast count of $\leq 5 \%$ with recovery of all the 3 cell lines in the peripheral blood. CR with incomplete count recovery, defined as no evidence of persistent disease but an incomplete recovery of blood counts, occurs frequently and is associated with a worse prognosis compared with patients with normal count recovery. In contrast, a small percentage of patients have an increased platelet count at the end of the month after initiation of induction chemotherapy, and the clinical significance of this has yet to be investigated. Our hypothesis was that CR increased platelet count at day 30 translated to a superior survival rate compared with $\mathrm{CR}$ with subnormal or normal platelet counts because of a potentially rapid recovery of the bone marrow after induction chemotherapy.
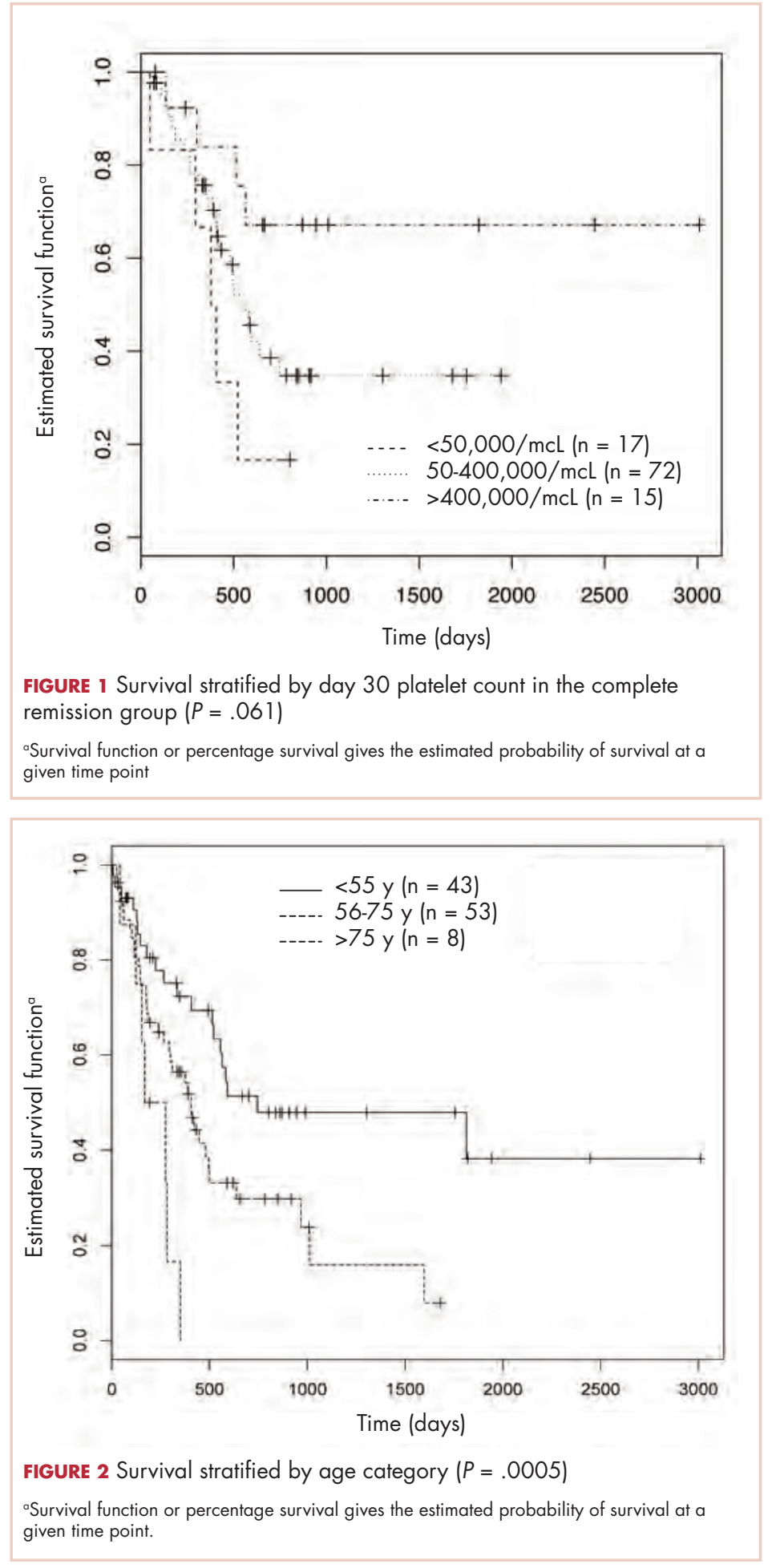

\section{Materials and methods}

A total of 104 patients diagnosed with AML were considered from the leukemia database registry of Georgia Regents University. Institutional review board approval was obtained prior to reviewing records. Adult AML patients ( $\geq 18$ years) who survived at least 30 days after initiation of 


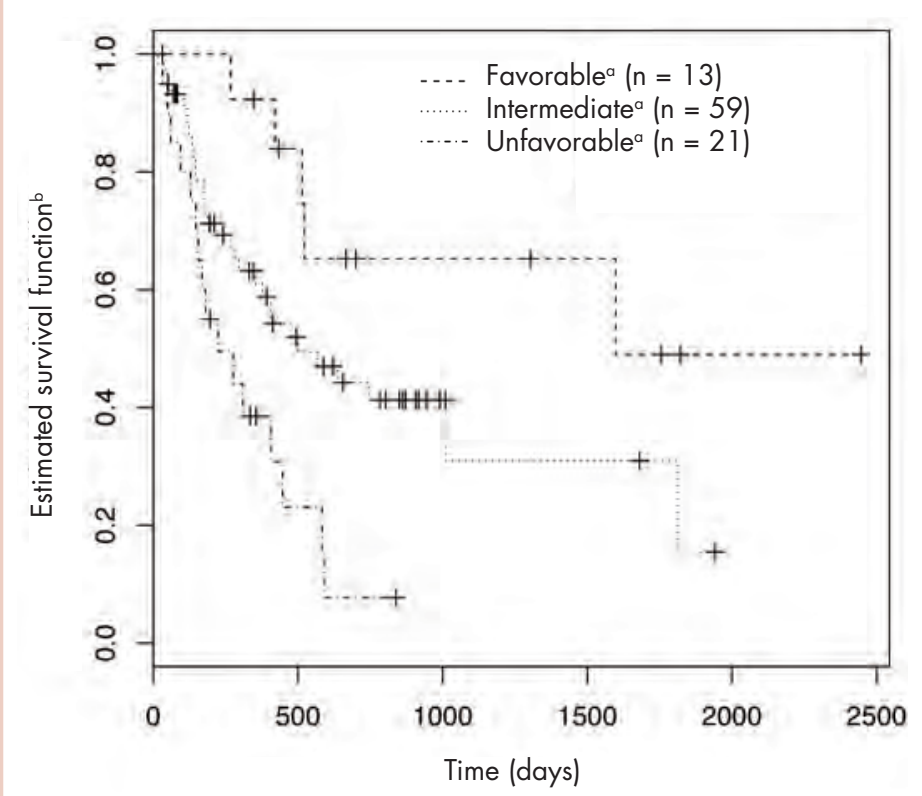

FIGURE 3 Survival stratified by risk categorya (based on cytogenetics/ molecular studies, $P=.0014$ )

aRisk categories as per the European LeukemiaNet recommendations ${ }^{8}$ are Favorable: $\dagger(8 ; 21)(q 22 ; q 22) ; R U N X 1-R U N X 1 T 1$; inv (16)(p13.1q22) or $t(16 ; 16)(p 13.1 ; q 22)$;

CBFB-MYHI 1, mutated NPM1 without FLT3-ITD, mutated CEBPA. Intermediate: mutated NPM1 and FLT3-ITD (normal karyotype); wild type NPM1 and FLT3-ITD (normal karyotype); wild type NPMI without FLT3-ITD (normal karyotype); $1(9 ; 11)(\mathrm{p} 22 ; \mathrm{q} 23)$; MLLT3-MLL; cytogenetic abnormalities not classified as favorable or unfavorable. Unfavorable: inv (3)(q2 1q26.2) or $\mathrm{t}(3: 3)(\mathrm{q} 21 ; \mathrm{q} 26.2)$; RPN1-EVI1 $\mathrm{t}(6 ; 9)(\mathrm{p} 23 ; \mathrm{q} 34)$; DEK-NUP2 14; t(v; 11$)(v ; q 23)$; MLL rearranged -5 or del $(5 q) ;-7 ;$ abnl(17p); complex karyotype. bSurvival function or percentage survival gives the estimated probability of survival at a given time point.

first cycle of induction chemotherapy with the cytarabine plus anthracycline (doxorubicin or daunorubicin) combination, or $7+3$ regimen, were included in the analysis. Untreated patients $(n=18)$ and patients who underwent platelet transfusions during days 25-35 after the first cycle of induction were excluded.

Patient characteristics such as age; sex; race; risk category based on cytogenetics/mutation analysis as per the European LeukemiaNet recommendations ${ }^{8}$ (Figure 3); culture-proven infections ( $\leq 1$ month before, during, and $\leq 6$ months after induction therapy); remission status (bone marrow blast cell percentage of $\leq 5$ ) at day 30 (bone marrow biopsy results were considered during days 25-35 after first cycle of induction chemotherapy); and whether allogeneic stem-cell transplantation (SCT) was performed or not, were recorded (Table 1). In addition, platelet count at day 0 (day of initiation of first cycle of induction chemotherapy); highest platelet count during days 25-35 (designated as day 30 platelet count); day $14+$ plasma cell count (average plasma cell percentage at days 14-21).

Overall survival (OS) was noted and survival estimates were calculated using Kaplan-Meir analysis. Patients from the complete remission group were divided into 3 groups: platelet count $<50,000 / \mathrm{mcL}(\mathrm{n}=17), 51,000-400,000 / \mathrm{mcL}$
( $\mathrm{n}=72)$, and $>400,000 / \mathrm{mcL}(\mathrm{n}=15)$; and their survival curves were compared (Figure 1). Survival was also stratified for age, sex, race, and risk (Figures 2, 3). A multivariate analysis was conducted for the following factors: age; sex; culture-proven infections, allogeneic SCT performed or not; day 30 platelet count; day 14+ plasma cell count; and induction chemotherapy considered or not (Table 2). Statistical analysis was done using Statistical Analysis Software (SAS edition 9.4).

\section{Results}

Mean follow-up period for the study was 168 months, and mean survival was 42 months. Mean age of the patients was 55.8 years. Age was grouped into 3 ranges: $<55$ years $(\mathrm{n}=$ $43)$; 56-75 years $(\mathrm{n}=53)$; and $>75$ years $(\mathrm{n}=8)$. KaplanMeir survival analysis was significant for higher survival in the $<55$-year age group, followed by the 56-75-year group and then the $>75$-year group (Figure 2, $P=.0005$ ). Sex distribution was fairly even, with 54 men and 50 women participating. No significant difference in OS was found between the sexes $(P=.839)$. The majority of the patients were white $(\mathrm{n}=65)$, followed by African-American $(\mathrm{n}=$ $35)$, and Hispanic ( $n=3) ; 1$ patient's race could not be ascertained from the records. No racial differences in survival were apparent $(P=.287)$. Based on the European LeukemiaNet's recommended risk categories, the favorable risk group had 13 patients; the intermediate and adverse risk groups had 59 and 21 patients, respectively; and 11 patients could not be categorized from records. As expected, OS was highest in the favorable group, followed by intermediate and poor risk groups respectively $(P=.0014$, Figure 3$)$. This difference was also highlighted in the multivariate analysis with poor risk category showing a higher odds ratio of 4.99 (95\% confidence index [CI], $1.53,16.32 ; P=.008)$. Of the 104 patients, 63 achieved $\mathrm{CR}$, defined as reduction in bone marrow blast cells to $\leq 5 \%$ after first induction cycle at the end of 1 month after diagnosis; 30 did not achieve CR; and status could not be ascertained from the records for 11 patients. In all, 16 of 104 patients underwent an allogeneic SCT. The average day 30 platelet count in the group receiving SCT was 216,000/ mcL and average OS was 745 days. As expected, allogeneic SCT had a strong effect on OS, with patients who did not undergo it having an odds ratio of 8.7 (95\% CI, 2.36, 32.07; $P=.0007)$.

Infections $\leq 1$ month before and during the first cycle of induction were not significant prognostic indicators of OS. Of the 104 patients, 24 had culture-proven infection during induction chemotherapy (group 1), 22 had infection $\leq 6$ months after induction chemotherapy (group 2), and 58 either had infections $\leq 6$ months before or had no infections recorded during those time frames (group 3). In group 1, average day 30 platelet count was $186 / \mathrm{mcL}$ and OS was 524 days; in group 2 , the numbers were $251 / \mathrm{mcL}$ and 395 


\begin{tabular}{|c|c|c|c|}
\hline Variable & Odds risk & $95 \% \mathrm{Cl}$ & $P$ value \\
\hline \multicolumn{4}{|l|}{ Age, $y$} \\
\hline$\leq 55$ & 1 & - & - \\
\hline $56-75$ & 1.54 & $0.7-3.40$ & 0.284 \\
\hline$\geq 75$ & 2.70 & $0.67-10.89$ & 0.163 \\
\hline \multicolumn{4}{|l|}{ Sex } \\
\hline Male & - & - & - \\
\hline Female & 0.88 & $0.43-1.79$ & 0.717 \\
\hline \multicolumn{4}{|l|}{ Culture-proven infection ${ }^{a}$} \\
\hline$\leq 1$ month before induction & 2.64 & $0.23-30.61$ & 0.438 \\
\hline During induction ${ }^{b}$ & 1.89 & $0.75-4.76$ & 0.178 \\
\hline$\leq 6$ months after induction & 4.20 & $1.83-9.67$ & 0.0007 \\
\hline \multicolumn{4}{|l|}{ Allogenic SCT } \\
\hline Yes & 1 & - & - \\
\hline No & 8.7 & $2.36-32.07$ & 0.0012 \\
\hline \multicolumn{4}{|l|}{ Remission } \\
\hline Yes & 1.22 & $0.53-2.80$ & 0.646 \\
\hline No & 4.49 & $1.17-17.21$ & 0.028 \\
\hline \multicolumn{4}{|l|}{ Day 30 platelet count ${ }^{c}$} \\
\hline$<50,000 / \mathrm{mcL}$ & 1 & - & - \\
\hline $50,000-400,000 / \mathrm{mcL}$ & 0.58 & $0.24-1.37$ & 0.2148 \\
\hline$>400,000 / \mathrm{mcL}$ & 0.22 & $0.06-0.83$ & 0.026 \\
\hline \multicolumn{4}{|c|}{ Day $14+$ plasma cell percentage ${ }^{d}$} \\
\hline 10 & 0.53 & $0.25-1.14$ & 0.106 \\
\hline$>10$ & 0.70 & $0.29-1.67$ & 0.422 \\
\hline \multicolumn{4}{|l|}{ Risk $^{e}$} \\
\hline Favorable & 1 & - & - \\
\hline Intermediate & 2.00 & $0.65-6.15$ & 0.225 \\
\hline Adverse & 4.99 & $1.53-16.32$ & 0.008 \\
\hline
\end{tabular}

days, respectively; and in group 3, they were $244 / \mathrm{mcL}$ and 537 days, respectively. This data suggests that infections were likely not responsible for upregulating platelet production. In addition, there was not a strong correlation between day 0 platelet count (platelet count at diagnosis and before the start of first cycle of induction chemotherapy) and day 0 platelet count $(\mathrm{r}=0.1891, P=.033)$.

Kaplan-Meir survival curves for the different platelet groups showed statistical significance using the log-rank test $(P=.005)$. This difference remained significant $(P=.012)$ after performing a multivariate analysis using the Cox Proportional Hazards Model. There was a similar trend in the CR group, $(P=.061, \log$-rank test, Figure 2$)$. Furthermore, on multivariate analysis, the $>400,000 / \mathrm{mcL}$ platelet group showed a significant lower odds ratio compared with the other groups $(0.22 ; 95 \% \mathrm{CI}, 0.06,0.83 ; P=.026)$, indicating a better OS. Of the 104 patients, 28 underwent relapse after the first induction cycle, 25 stayed in remission, and the status of 51 patients could not be reliably ascertained from the medical records. A similar trend was observed in the relapse-free survival among the platelet survival groups (354.6 days for the $<50,000 / \mathrm{mcL}$ group ( $\mathrm{n}=5$ ); 372 days for the $50,000-400,000 / \mathrm{mcL}$ group $(n=34)$, and 805.07 days $(n=14)$ for the $>400,000 / \mathrm{mcL}$ group), see Table 2 .

\section{Discussion}

Complete remission in acute myeloid leukemia is defined as having a blast count of $\leq 5 \%$ in a bone marrow peripheral smear, platelet $(>100,000 / \mathrm{mcL})$ and neutrophil recovery $\left(>1.0 \times 10^{9} / \mathrm{mcL}\right)$ and no extramedullary involvement. In HOVON-SAKK co-operative group studies, the median time to remission was about 32 days. ${ }^{9}$ However, a small subset of patients with blast counts of $\leq 5 \%$ but incomplete platelet recovery at the end of 1 month of induction therapy showed poor outcomes. ${ }^{10}$ This exclusive subset, known as CR with incomplete platelet recovery, fulfilled all the criteria of remission except that platelet counts were less than $100,000 / \mathrm{mcL}$, provided that patients were not depen- 
dent on platelet transfusions. On the one hand, although $\mathrm{CR}$ with incomplete platelet recovery shows poor outcomes when compared with $\mathrm{CR}$, it is a distinct subcategory in itself and shows a clinically meaningful response to drugs as it correlates with better outcomes when compared with resistant disease. ${ }^{10}$ On the other hand, there is another small subset of patients who achieve $\mathrm{CR}$ with blasts count $\leq 5 \%$ and who have much higher platelet counts $(>400,000$ / $\mathrm{mcL}$ ). Our data suggests that the latter have better outcomes than those with $\mathrm{CR}$ with incomplete or normal platelet recovery. We theorize that an increased platelet count at the end of 1 month after initiation of induction chemotherapy is indicative of a more robust bone marrow recovery and response to induction chemotherapy, and this translates to an increased survival. The mechanism certainly needs to be further elucidated.

Bolwell and colleagues assessed the prognostic importance of day 100 platelet count after allogeneic SCT in patients with AML. They looked at 107 patients who survived at least 100 days following an ablative allogeneic SCT and found the higher platelet count to be a strong predictor of OS. ${ }^{11} \mathrm{Al}$-Shugair and colleagues reported that day 14+ plasma cells in the bone marrow aspirates after induction chemotherapy correlated positively with the presence of residual leukemic blast cells, although they found no correlation with OS. ${ }^{12}$ The mechanism for this trend was thought to be secondary to an increased interleukin-6 (IL-6) secretion from leukemic blast cells stimulating plasma cell production. Antecedent infections and/ or other inflammatory processes are postulated to increase plasma cell production. Also, they found higher plasma cell counts in patients with antecedent or concurrent infection and/or inflammation compared with those without (18.1 vs $10.7 \%, P=.039) .{ }^{12}$ It is debatable at this time whether the same translates for platelet counts. In our multivariate analysis, we did not find day 14+ plasma cell count to be an independent predictor of survival.

\section{References}

1. American Cancer Society: Cancer Facts and Figures. Atlanta, Ga: American Cancer Society. 2014.

2. Rowe JM, Tallman MS. How I treat acute myeloid leukemia. Blood. 2010;116:3147-3156.

3. Conway O'Brien E, Prideaux S, Chevassut T. The epigenetic landscape of acute myeloid leukemia. http://dx.doi.org/10.1155/2014/103175. Published March 23, 2014. Accessed January 22, 2016.

4. Foon KA, Zighelboim J, Yale C, Gale RP. Intensive chemotherapy is the treatment of choice for elderly patients with acute myelogenous leukemia. Blood.1981;58:467-470.

5. Jin J, Chen J, Suo S et al. Low-dose cytarabine, aclarubicin and granulocyte colony-stimulating factor priming regimen versus idarubicin plus cytarabine regimen as induction therapy for older patients with acute myeloid leukemia. Leuk Lymphoma. 2015;56:1691-1697.

6. Lowenberg B. Treatment of the elderly patient with acute myeloid leukaemia. Baillieres Clin Haematol. 1996;9:147-159.

7. Kim SJ, Cheong JW, Kim DY, et al. Role of induction and consolidation chemotherapy in elderly acute myeloid leukemia patients. Int J Hematol. 2014;100:141-51.
Several prognostic factors have been identified for patients with AML. Historically, age was recognized as one of the most important prognostic factors. It is well known that older age group is associated with adverse outcomes because of poorer performance status and unfavorable cytogenetics. ${ }^{13}$ Our data are consistent with this and show an adverse prognosis with increasing age (Figure 2). AML is known to be a clinically heterogeneous disease and blast cell morphology is another important determinant of prognosis. ${ }^{14}$ Cytogenetics remains the most important prognostic factor, with the disease characterized as favorable, intermediate, or poor risk. ${ }^{12,14-17}$ Our data was consistent with the established risk factors, with OS showing the expected trend in each of these groups $(P=.0014)$.

Another potential confounding factor affecting day 30 platelet count is infections. Infections have been postulated to cause IL-6 upregulation and stimulation of thrombopoeitin production in the liver, which can facilitate platelet production. ${ }^{17,18}$ We evaluated for an occurrence of infections $\leq 1$ month before, during, and $\leq 6$ months after induction chemotherapy. The group with infections during induction chemotherapy had lower average platelet counts, suggesting that infections likely did not falsely upregulate platelet counts. Infections during induction chemotherapy (days 0-30) was not a statistically significant independent predictor of survival in our analysis (OR, 1.89; 95\% CI, 0.75-4.76; $P=.178)$.

Future research should include studies to elucidate underlying molecular pathophysiology to identify novel therapeutic targets. Subcategorizing CR to incomplete, complete, and superior platelet recovery may help in better delineation of efficacy of future investigational agents.

In conclusion, $\mathrm{CR}$ with superior platelet count is a unique subcategory in itself and should be further categorized into $\mathrm{CR}$ with incomplete, normal, and superior platelet count to better characterize response to induction chemotherapy.

8. Dohner H, Estey EH, Amadori S et al. Diagnosis and management of acute myeloid leukemia in adults: recommendations from an international expert panel, on behalf of the European LeukemiaNet. Blood. 2010;115:453-474.

9. de Greef GE, van Putten WL, Boogaerts M, et al. Criteria for defining a complete remission in acute myeloid leukaemia revisited. An analysis of patients treated in HOVON-SAKK co-operative group studies. Br J Haematol. 2005;128:184-191.

10. Walter RB, Kantarjian HM, Huang X, et al. Effect of complete remission and responses less than complete remission on survival in acute myeloid leukemia: a combined Eastern Cooperative Oncology Group, Southwest Oncology Group, and MD Anderson Cancer Center Study. J Clin Oncol. 2010;28:1766-7111.

11. Bolwell B, Pohlman B, Sobecks R, et al. Prognostic importance of the platelet count 100 days post allogeneic bone marrow transplant. Bone Marrow Transplant. 2004;33:419-423.

12. Al-Shughair N, Al-Dawsari G, Gyger M, Mohamed G, Roberts G. Clinical significance of plasmacytosis in the day +14 bone marrow of patients with acute myeloid leukaemia undergoing induction chemo- 
therapy. J Clin Pathol. 2007;60:520-523.

13. Appelbaum FR, Gundacker H, Head DR, et al. Age and acute myeloid leukemia. Blood. 2006;107:3481-3485.

14. Foran JM. New prognostic markers in acute myeloid leukemia: perspective from the clinic. Hematology Am Soc Hematol Educ Program. 2010;2010:47-55.

15. Delaunay J, Vey N, Leblanc T, et al. Prognosis of inv(16)/t(16;16) acute myeloid leukemia (AML): a survey of 110 cases from the French AML Intergroup. Blood. 2003;102:462-469.

16. Grimwade D, Jamal R, Goulden N, Kempski H, Mastrangelo S, Veys P. Salvage of patients with acute promyelocytic leukaemia with residual disease following ABMT performed in second CR using alltrans retinoic acid. Br J Haematol. 1998;103:559-562.

17. Lu H, Jiang T, Li R, Wang S, Zhang Q, Zhao S. Bone marrow stromal cells transduced with a thrombopoietin, interleukin-6, and interleukin-11 syncretic gene induce cord mononuclear cells to generate platelets in vitro. Transfusion. 2015;55:176-186.

18. Chang M, Suen Y, Meng G et al. Differential mechanisms in the regulation of endogenous levels of thrombopoietin and interleukin-11 during thrombocytopenia: insight into the regulation of platelet production. Blood. 1996;88:3354-3362. 